\author{
Miguel Alcaide ${ }^{1,2 \times}$ \\ Lidia López ${ }^{2 \times}$ \\ Alessandro Tanferna ${ }^{2}$ \\ Julio Blas ${ }^{2}$ \\ Fabrizio Sergio ${ }^{2}$ \\ Fernando Hiraldo ${ }^{2}$ \\ ${ }^{1}$ Department of Organismic and \\ Evolutionary Biology, Harvard \\ University, Cambridge, MA, \\ USA \\ ${ }^{2}$ Estación Bioló gica de Doñana \\ (CSIC), Sevilla, Spain
}

Received November 21, 2009

Revised January 10, 2010

Accepted January 10, 2010

\section{Simultaneous analysis of multiple PCR amplicons enhances capillary SSCP discrimination of $\mathrm{MHC}$ alleles}

\begin{abstract}
Major histocompatibility complex (MHC) genotyping still remains one of the most challenging issues for evolutionary ecologists. To date, none of the proposed methods have proven to be perfect, and all provide both important pros and cons. Although denaturing capillary electrophoresis has become a popular alternative, allele identification commonly relies upon conformational polymorphisms of two single-stranded DNA molecules at the most. Using the MHC class II (b chain, exon 2) of the black kite (Aves: Accipitridae) as our model system, we show that the simultaneous analysis of overlapping PCR amplicons from the same target region substantially enhances allele discrimination. To cover this aim, we designed a multiplex PCR capable to generate four differentially sized and labeled amplicons from the same allele. Informative peaks to assist allele calling then fourfold those generated by the analysis of single PCR amplicons. Our approach proved successful to differentiate all the alleles (N 513 ) isolated from eight unrelated birds at a single optimal run temperature and electrophoretic conditions. In particular, we emphasize that this approach may constitute a straightforward and cost-effective alternative for the genotyping of single or duplicated MHC genes displaying low to moderate sets of divergent alleles.
\end{abstract}

\section{Keywords:}

Adaptive genetic variation / Black kite / Major histocompatibility complex class II / Major histocompatibility complex genotyping / Pathogen resistance
Genetic variation at the coding regions that conform the antigen-binding sites of Major histocompatibility complex (MHC) molecules has attracted the attention of evolutionary biologists because of its well-known role in sexual selection and pathogen resistance [1,2]. The survey of MHC diversity is however a challenging and time-consuming task, especially when locus specific typing protocols cannot be developed and species display both extensive genetic polymorphism and a high number of gene duplications. Molecular cloning and Sanger sequencing along with allele discrimination through denaturing gel electrophoresis have been the most widely employed methods. The advent of nextgeneration sequencing technologies is nonetheless revolutionizing MHC genotyping strategies (reviewed in [3]). Indirect approaches to MHC variation are considered more

Correspondence: Dr. Miguel Alcaide, Department of Organismic and Evolutionary Biology, Harvard University, 26 Oxford Street, 02128, Cambridge, MA, USA

E-mail: malcaide@oeb.harvard.edu

Fax: 11-617-495-5667

Abbreviations: DGGE, denaturing gradient gel electrophoresis; MHC, major histocompatibility complex straightforward and cost-effective than Sanger DNA-sequencing methods, and different alternatives such as denaturing gradient gel electrophoresis (DGGE), SSCP or reference strand-mediated conformational analyses (RSCA) have been applied to survey levels of MHC variation in different vertebrate species [3]. These approaches rely on the fact that single-stranded DNA molecules of the same length but different nucleotide sequence may have different folding properties. The secondary structure of single-stranded DNA molecules decisively affects their migration rate across a polymeric matrix exposed to an electromagnetic field. In particular, the possibility of adding fluorescent dyes to DNA molecules and resolving them on automated capillary electrophoretic systems has increased the popularity of SSCP and RSCA approaches [4, 5] over the use of laborious and toxic DGGE gel electrophoresis gels [6]. However, capillary electropheresis also requires the further combination of molecular cloning and Sanger sequencing to characterize individual alleles. Although denaturing gel electrophoresis is intended to distinguish single nucleotide substitutions, large repertoires of MHC alleles are sometimes difficult to resolve and demand for careful optimization.

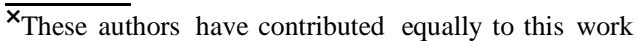


Despite the urgent need for designing straightforward and cost-effective methods to screen MHC variation, indirect approaches have focused exclusively on the analysis of single PCR amplicons encompassing the whole or part of the antigen-binding site. Thus, the information required for allele discrimination is restricted to two single-stranded DNA molecules at the most. Here, we tested whether the simultaneous analysis of different sized PCR amplicons from the same target region would represent a major improvement for allele discrimination. To cover this aim, we isolated polymorphic MHC class II loci (b chain, exon 2) in the black kite Milvus migrans (Aves: Accipitridae) and designed a multiplex PCR assay.
DNA extracts were obtained from eight unrelated adult black kites trapped in 2008 in Doñana National Park (southwestern Spain). Blood samples preserved in absolute ethanol were treated according to a HotSHOT protocol adapted from Truett et al. [7]. Blood was suspended in $100 \mathrm{~mL}$ of a solution containing $\mathrm{NaOH} 25 \mathrm{mM}$ plus EDTA $0.2 \mathrm{mM}$ and incubated at $951 \mathrm{C}$ for $15 \mathrm{~min}$. Reactions were neutralized by adding $100 \mathrm{~mL}$ of a $40 \mathrm{mM}$ Tris- $\mathrm{HCl}$ neutralization buffer. The second exon of MHC class II B genes was amplified using primers Acc2FC and Acc2RC following the PCR protocol and conditions described in [8]. PCR products were subsequently cleaned-up using Microcon centrifuge filters (Millipore) and cloned using

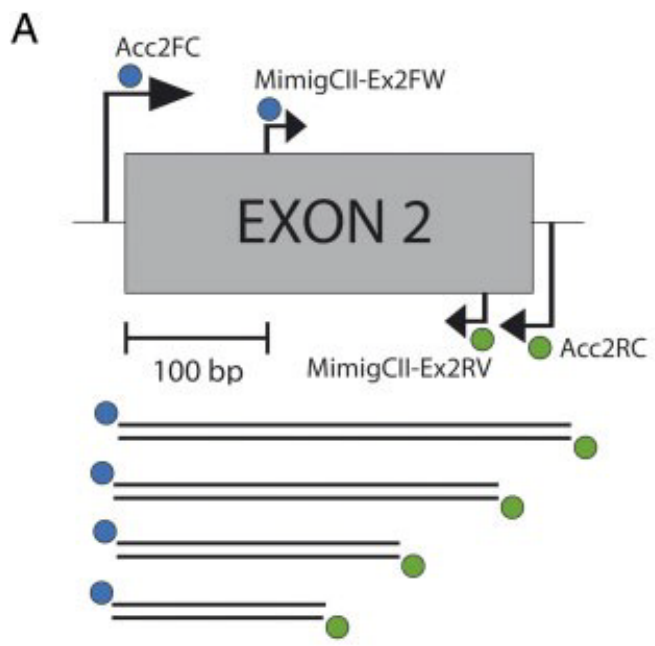

B

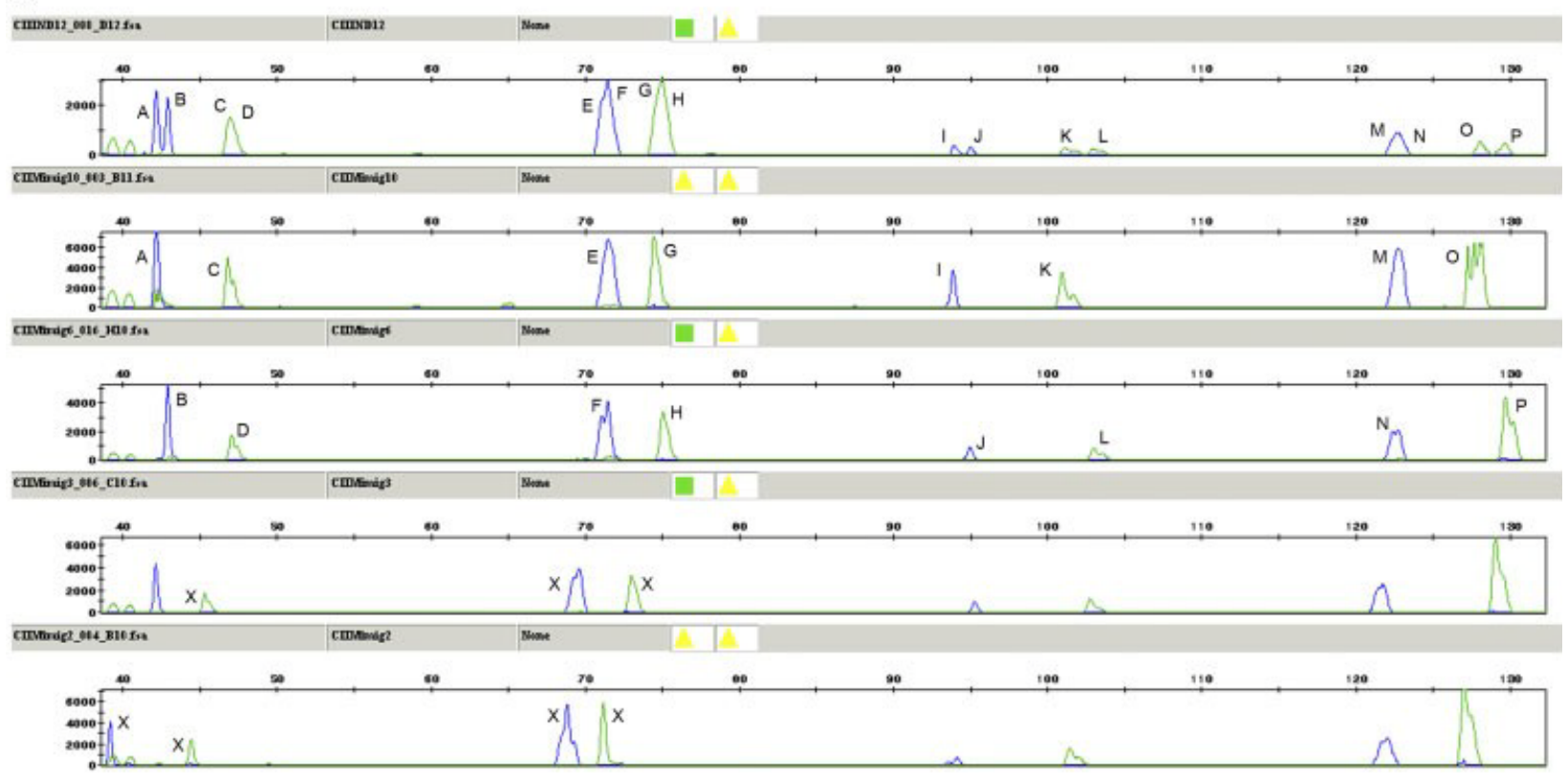

Figure 1. (A) Location of primers sitting on the exon 2 of MHC class II B genes and flanking introns. PCR using these primers generates 4 amplicons of different size. Colour dots represent different dyes: 6-FAM (blue) and VIC (green). (B) SSCP electrophoresis of multiplexed PCR-MHC class II amplicons. SSCP profiles composed of eight informative peaks are shown for four cloned alleles (Mimig 2, 3, 6 and 10). Above them, SSCP profile of one individual of known allelic composition (Mimig61Mimig10). Letters indicate the identity of different peaks. Peaks that exclude the presence of particular alleles within the displayed electropherogram are indicated by $\mathrm{X}$. 
the PGEM-T easy vector system II (Promega). Sixteen positive clones (i.e. those containing an insert of the expected size) were sequenced according to the Big Dye 1.1 Terminator technology. Labeled fragments were resolved using an ABI 3130xl automated sequencer (Applied Biosystems).

We isolated a maximum of four different alleles per individual and a total number of 13 class II alleles (Supporting Information Fig. S1). This finding, in agreement with data collected in other Accipitridae species [8], suggests the co-amplification of two gene duplications. Cloned alleles were aligned and edited using BioEdit 7.0.9 [9]. Polymorphism statistics calculated using the software DNAsp [10] revealed 42 segregating sites corresponding to 49 mutations, a nucleotide diversity p 50.074 and an average number of nucleotide differences between alleles k 5 19.11. Black kite MHC class II sequences have been deposited in GenBank (GenBank Acc. Nos. GU390260-72). Two internal primers were designed over conserved regions emerging from the alignment of the black kite class II data set. These primers were MimigCII-EX2F ( $5^{\circ}$-CACTTTGACAGCGATGTGG- $3^{0}$ ) and MimigCII-EX2RV (5 ${ }^{\circ}$-CACCTCGTAGTTGTGTCG-3 ${ }^{0}$ ). Our main goal was to design a multiplex PCR capable of generating four overlapping amplicons. PCR amplicons were intended to cover the entire optimal range size (100-300 bp, see Fig. 1) recommended for SSCP analysis [11]. Internal primers were multiplexed with primers Acc2FC and Acc2RC in a PCR reaction following the same protocol described in [8] but now containing $1 \mathrm{~mL}$ of DNA from those positive clones reporting different MHC alleles. At this stage, primers were fluorescently labeled with 6-FAM and VIC dyes (Fig. 1). PCR products were diluted 1:10 and denatured using Hi-Di formamide and heat during incubations of $3 \mathrm{~min}$ at 951C. Capillary electrophoresis on an ABI 3130xl Genetic Analyser (Applied Biosystems) was carried out using a polymeric matrix composed of 5\% CAP (Applied Biosystems), 10\% glycerol and $1 \times$ Genetic Analyzer running buffer. Samples were run at 181C, 261C and 331C, with $0.7 \mathrm{~mL}$ of GeneScan 500-LIZ size standard using the FragmentAnalysis36_POP4 run module. The room holding the sequencer was airconditioned to maintain the temperature below the run temperature. The electrophoretical mobility of DNA fragments marked with different dyes was analyzed using the software GenMapper software ver 3.7 (Applied Biosystems).

Run temperature was the most critical parameter influencing the quality of SSCP profiles. Thus, while run temperatures of 18 and 261C generated complex peaks, peaks were sharpest when running samples at 331C (Supporting Information Fig. S2). At this temperature, we successfully obtained eight informative SSCP peaks for each particular MHC allele we cloned (Fig. 1, Table 1). SSCP profiles seemed to be reproducible while maintaining non-denaturing electrophoretic conditions and parameters. Furthermore, individual allele profiles could be recognized after conducting multiplex PCR experiments on DNA extracts from particular birds of known MHC genotypes (Fig. 1).
Table 1. Summary of the emitted fluorescence for each particular dye during multiplex PCR-SSCP analysis of cloned MHC class II alleles ${ }^{\text {a) }}$

\begin{tabular}{lllll}
\hline & 6-FAM & 6 -FAM & 6-FAM & 6-FAM \\
& VIC & VIC & VIC & VIC \\
\hline Mimig12 & 114.65 & $85.85-86.57$ & 67.86 & $36.92-37-73$ \\
& $121.47-122.04$ & $94.83-95.48$ & 71.47 & $43.78-44.22$ \\
Mimig11 & 117.97 & 90.51 & $66.69-67.13$ & $37.69-37.96$ \\
& 123.80 & $98.25-9865$ & 70.83 & $44.01-44.46$ \\
Mimig4 & $120.5-120.75$ & 92.14 & 68.17 & 37.81 \\
& $127.65-128.17$ & $102.16-102.77$ & 71.24 & $44.47-44.91$ \\
Mimig8 & 121.45 & $92.90-93.60$ & 70.43 & $41.34-42.06$ \\
& $126.43-127.02$ & 100.68 & 73.61 & 46.61 \\
Mimig9 & 121.45 & 93.35 & 70.75 & 42.09 \\
& 129.60 & 102.75 & 73.54 & 46.60 \\
Mimig3 & $121.5-121.76$ & 95.23 & $69.34-69.60$ & 42.13 \\
& 129.03 & 102.75 & 72.98 & 45.29 \\
Mimig2 & $121.69-122.07$ & 94.12 & 68.80 & 39.14 \\
& 127.00 & 101.45 & 71.17 & 44.43 \\
Mimig6 & $122.37-122.75$ & 94.95 & $71.04-71.43$ & 42.89 \\
& 129.72 & 102.97 & 75.03 & 47.04 \\
Mimig10 & 122.75 & $93.14-93.86$ & 71.43 & 42.13 \\
& 128.03 & 100.9 & 74.46 & 46.79 \\
Mimig5 & 123.27 & 96.04 & 70.25 & $41.34-42.06$ \\
& $129.48-129.97$ & $103.68-104.37$ & 73.13 & 45.72 \\
Mimig7 & 123.15 & 96.04 & 70.25 & $41.34-42.06$ \\
& $129.22-129.68$ & $103.43-104.13$ & 73.13 & 45.72 \\
Mimig13 & $123.96-124.57$ & $94.04-94.76$ & $71.13-71.50$ & $41.50-42.29$ \\
& $130.15-130.81$ & $103.88-104.65$ & 74.62 & 46.90 \\
Mimig1 & $125.4-125.85$ & $97.99-98.56$ & 71.23 & 43.45 \\
& $129.83-130.42$ & $102.87-103.49$ & 74.00 & 46.25 \\
\hline & & & & \\
\hline
\end{tabular}

a) SSCP peaks exclusively reported for particular alleles and clearly differentiated from the peaks displayed by other alleles are indicated in bold. Alleles are organized regarding one of the largest, labeled and single stranded DNA molecules. Peaks positions were determined using GeneMapper version 3.7.

We therefore encourage the simultaneous analysis of overlapping MHC amplicons to increase the sensitivity of SSCP approaches. Having said this, there are some important caveats that should be accounted for if multiplex-PCR approaches are going to be systematically performed on DNA extracts rather than on cloned alleles. The co-amplification of overlapping PCR amplicons from the same or duplicated loci may substantially promote the formation of chimeric sequences. Although some authors have proposed efficient strategies to minimize the effects of in vitro recombination [12], we strongly recommend independent PCR reactions to characterize individual MHC profiles. While this procedure would imply a considerable increase in sample manipulation and overall costs, the multiplex of primers targeting to non-homologous MHC loci can be, on the contrary, safely accomplished. For instance, primers suitable for the amplification of MHC class I genes in raptors have become recently available [13]. MHC class I and class II primers, previously labeled with different dyes, could therefore be multiplexed in the black kite and 
amplicons can be simultaneously resolved via automated capillary electrophoresis.

In conclusion, we describe a straightforward and costeffective alternative for the screening of MHC variation. According to our results, this method would be ideal for those species and/or populations displaying low to moderate sets of relatively divergent MHC alleles. Such kinds of patterns have been predominantly documented, for instance, across island populations of vertebrate species [14-16].

The authors thank J. M. Giralt for help in the field. Part of this study was funded by the research projects CGL2008-01781 of the Ministerio de Ciencia e Innovación, JA-58 of the Consejería de Medio Ambiente de la Junta de Andalucía and by the Excellence Project RNM 1790 and RNM 03822 of the Junta de Andalucía.

The authors have declared no conflict of interest.

\section{References}

[1] Sommer, S., Front. Zool. 2005, 2, 16.

[2] Piertney, S. B., Oliver, M. K., Heredity 2006, 96, 7-21.

[3] Babik, W., Mol. Ecol. Res. 2010, 10, 237-251.
[4] Bryja, J., Galan, M., Charbonnel, N., Cosson, J. F., Mol. Ecol. Res. 2005, 5, 173-176.

[5] Lenz, T. L., Eizaguirre, C., Becker, S., Reusch, T. B. H., Biomed. hromatogr. Evol. Biol. 2009, 9, 57.

[6] Westerdahl, H., Wittzell, H., von Schantz, T., Bensch, S., Heredity 2004, 92, 534-542.

[7] Truett, G. E., Walker, J. A., Truett, A. A., Mynatt, R. L., Heeger, P., Warman, M., Biotechniques 2000, 29, 52-54.

[8] Alcaide, M., Edwards, S. V., Negro, J. J., J. Mol. Evol. 2007, 65, 541-554.

[9] Hall, T. A., Nucleic Acids Symp. Ser. 1999, 41, 95-98.

[10] Rozas, J., Sánchez-DelBarrio, J. C., Messeguer, X., Rozas, R., Bioinformatics 2003, 19, 2496-2497.

[11] Hayashi, K., Genet. Anal. Tech. Appl. 1992, 9, 73-79.

[12] Lenz, T. L., Becker, S., Gene 2008, 427, 117-123.

[13] Alcaide, M., Edwards, S. V., Cadahía, L., Negro, J. J., Conserv. Genet. 2009, 10, 1349-1355.

[14] Aguilar, A., Roemer, G., Debenham, S., Binns, M., Garcelon, D., Wayne, R. K., Proc. Natl. Acad. Sci. USA 2004, 101, 3490-3494.

[15] Richardson, D., Westerdahl, H., Mol. Ecol. 2003, 12, 3523-3529.

[16] Alcaide, M., Lemus, J. A., Blanco, G., Serrano, D., Negro, J. J., Rodríguez, A., García-Montijano, M., Mol. Ecol. 2010, 19, 691-705. 